\title{
Oral perception of the temperature of liquids
}

\author{
BARRY G. GREEN \\ Monell Chemical Senses Center, Philadelphia, Pennsylvania
}

\begin{abstract}
The ability of humans to sense the thermal characteristics of liquids held in the mouth was measured in two experiments. It was found in the first experiment that warming was sensed more strongly than cooling when the suprathreshold oral response to warm and cold liquids was compared in terms of the heat transferred between the oral mucosa and the liquid. The second experiment extended the investigation to threshold and again found warming to be perceived more readily than cooling. These results contrast with earlier findings from studies of local thermal sensitivity that indicated the mouth was more uniformly sensitive to cooling than to warming. It is proposed that the occurrence of spatial summation in the warmth sense and the relatively high resting temperature of the mouth predispose the oral cavity to be sensitive to increases in skin temperature.
\end{abstract}

Thermal sensations pervade the experience of drinking. The relatively high resting temperature of the mouth (ca. $36^{\circ} \mathrm{C}$ ) dictates that liquids drunk at all but the hottest ambient temperatures will cool the oral tissues, and the modern practice of cooking and refrigerating foods has led to a proliferation of flavored beverages served at temperatures far above (e.g., $50^{\circ}-60^{\circ} \mathrm{C}$ ) and far below (e.g., $0^{\circ}-10^{\circ} \mathrm{C}$ ) oral temperature. Although the saliency of oral thermal sensations suggests that the mouth is well equipped to sense the temperature of these liquids, psychophysical measurements of local responsiveness to both punctate and larger contact thermal stimulators have raised questions about the temperature sensitivity of the oral mucosa. Early maps of temperature-sensitive spots in the mouth implied that some areas (e.g., the medial and posterior surface of the tongue) were virtually insensible to warmth (Hirsch \& Schriever, 1929), and that the oral cavity was more uniformly sensitive to cooling than to warming. A more recent study that employed larger metallic stimulators corroborated the spatial heterogeneity of oral warmth responsiveness, but also discovered that the dorsum of the tongue possessed a moderate sensitivity to warmth when stimulus size exceeded punctiform dimensions (Green, 1984). Overall, however, the oral tissue indeed seemed better equipped to sense cooling than warming.

In accordance with these results, I undertook the present study with the expectation that cool liquids would be more acutely perceived than warm liquids. Two experiments demonstrated that this hypothesis was incorrect. When measured in terms of either threshold or perceived intensity, the thermal senses in the oral cavity signal the presence of higher temperatures more readily and more

This research was supported by Grant NS 20577 from the National Institutes of Health. The author thanks Barbara Gelhard for her help in running the experiments and analyzing the data. The author's mailing address is: Monell Chemical Senses Center, 3500 Market Street, Philadelphia, PA 19104. strongly than they signal the presence of lower temperatures.

\section{EXPERIMENT 1}

This experiment compared oral responsiveness to suprathreshold levels of warming and cooling in terms of both the temperature of the liquid and the heat transferred between the liquid and the oral structures. Heat transfer was estimated by measuring changes in the temperature of the liquid that occurred during sipping. The stimuli included decaffeinated coffee, in addition to deionized water, to determine whether the taste of a liquid and the temperature (hot or cold) at which it is normally experienced affect oral perception of thermal intensity.

\section{Method}

Subjects. Sixteen naive observers $(8$ men and 8 women, average age 24.4 years) were paid to participate in the experiment.

Apparatus and Procedure. The experiment included two kinds of liquid stimuli: $20-\mathrm{ml}$ samples of deionized water and $20-\mathrm{ml} \mathrm{sam}$ ples of a solution of $0.7 \%$ freeze-dried decaffeinated coffee and deionized water. The samples, which were held in glass vials, were either warmed to $37^{\circ}, 39^{\circ}, 41^{\circ}, 43^{\circ}, 45^{\circ}$, and $47^{\circ} \mathrm{C}$ or cooled to $30^{\circ}, 28^{\circ}, 24^{\circ}, 20^{\circ}, 16.5^{\circ}$, and $10^{\circ} \mathrm{C}$ in constant-temperature water baths. Bath temperatures were monitored with thermocouples connected through a switch box to a digital thermocouple thermometer.

Subjects held the vials containing the liquid stimuli in their gloved hands and sipped the liquids as rapidly as possible through a $12-\mathrm{cm}$ Teflon tube $(0.5 \mathrm{~cm}$ i.d.). The tube eliminated contact between the liquid and the vermilion border of the lip, which is known to be more sensitive than at least some areas of the oral mucosa (Green, 1984). The liquid was retained in the mouth without swishing for $3 \mathrm{sec}$ (timed by an audible metronome) and was then expectorated through an inverted plastic funnel (i.e., by placing the lips around the funnel's narrow spout and ejecting the liquid through it). A funnel was used, rather than the Teflon tube, because its larger diameter permitted more rapid expectoration.

A calibration procedure was devised to obtain estimates of heat transfer during sipping. A microthermocouple (Type T, $40 \mathrm{ga}$.) affixed to the outside of the Teflon sipping tube at its base provided a reading of solution temperature within $\pm 0.1^{\circ} \mathrm{C}$ at the moment 
of oral intake. Aliquots were sipped and retained in the mouth exactly as they were in the experiment itself and then were expectorated through the Teflon tube, where water temperature was again read. (The water was returned through the tube into the glass container from which it was drunk in order to minimize the influence of environmental temperature on the measurements.) The difference in temperature $(\Delta T)$ between the water before intake and the water after expectoration was used to calculate heat transfer (in joules, where 1 calorie $=4.186 \mathrm{~J}$ ) during sipping. Two subjects, a male and a female, sipped the 12 water temperatures five times each on 3 different days to produce a total of 30 measurements of temperature change per stimulus.

Because only six water baths were available for testing, it was necessary to run the warm and cold temperatures on separate days. To permit direct comparisons between each subject's magnitude estimates of warmth and cold, a magnitude matching paradigm was devised in which subjects began and ended each session by judging the loudness of a series of $1000-\mathrm{Hz}$ tones. A series consisted of tones at $30,40,50,60,70$, and $80 \mathrm{~dB} \mathrm{HL}$, delivered through headphones and lasting approximately $0.5 \mathrm{sec}$ each, administered twice each in random sequence. Subjects were instructed in the method of magnitude estimation and were told to practice by making judgments in response to distances the experimenter produced between her hands. The first three estimates of tonal intensity were also considered practice and were discarded. After the first series of tones, subjects were instructed to assign to the perceived intensities of oral thermal sensations numbers that reflected their strength relative to the loudness of the tones.

Half of the subjects were exposed to cold temperatures first and half to warm temperatures first. Trials were blocked within a session according to liquid type (coffee or water), with the order of presentation counterbalanced across subjects. Three judgments of perceived thermal intensity were made at each of the six warm and six cool temperatures. The magnitude estimates of warmth and cold (a total of 48 for each stimulus) were standardized to the tone judgments in the following way: For each subject, the geometric mean of the tone judgments made on the day judgments of cold were made was divided by the geometric mean of the tone judgments made on the day judgments of warmth were made. The resulting quotient was multiplied by the mean of the judgments of warmth. The overall standardization factor (averaged across all subjects) turned out to be 1.06 , meaning the average judgments of tone intensity differed by only $6 \%$ between warm and cold sessions

\section{Results and Discussion}

The geometric means of the standardized data for warmth and cold are plotted in Figures 1 and 2 as a function of the initial temperatures of the liquids. The most obvious result is that the perceived thermal intensity of

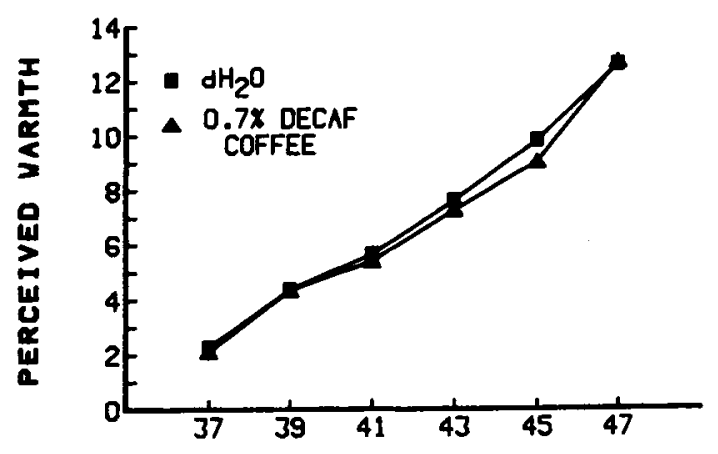

LIOUID TEMPERATURE (C०)

Figure 1. Perceived warmth as a function of the initial temperature of $20-\mathrm{ml}$ aliquots of deionized water or solutions of decaffeinated coffee and deionized water.

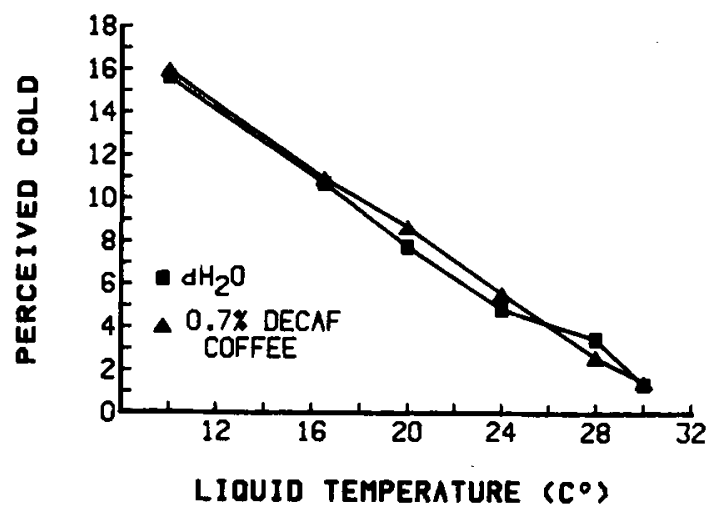

Figure 2. Same as Figure 1, for cold aliquots.

a liquid is unaffected by the presence of a taste or by the temperature at which the liquid is normally drunk. Coffee, usually drunk warm or hot, and water, usually drunk cool or cold, produced virtually identical perceived magnitudes throughout the range of temperatures.

The functions for both warmth and cold are well described by linear equations ( $r \leq 0.99$ ) having different slopes. These slopes indicate that sensations of warmth grow more rapidly as solution temperature increases (slope $=0.94$ ) than do sensations of cold as solution temperature decreases (slope $=-.70$ ). This implies that the sensitivity to temperature change in the whole mouth may be greater during warming than during cooling. However, the slopes of the psychophysical functions alone provide no information about the relative strength of the thermal sensations that would be evoked by "equivalent" thermal stimuli. That is, sensations of cold may neveretheless be more intense when a particular quantity of heat is transferred from the mucosa than are sensations of warmth when the same quantity of heat is transferred to the mucosa. To determine whether this was the case, the data of Figures 1 and 2 were replotted to show perceived thermal intensity as a function of the amount of heat estimated to have been transferred to and from the oral tissues. Figure 3 clearly shows that movement of heat into the oral tissues (warming) is perceived more strongly than movement of heat out of the oral tissues (cooling). To produce thermal sensations of equal intensity, heat transfer must be more than twice as great during cooling than during warming. It should be noted, however, that depiction of the data in terms of heat energy does not imply that transfer of heat is necessarily the critical stimulus dimension for oral thermal sensation. ${ }^{1}$ Estimated heat transfer provides a metric of the intensity of the overall thermal stimulus and has relevance to the primary function of the thermal senses, which is to assist in the regulation of heat flow between the body and the environment.

\section{EXPERIMENT 2}

The data of Figures 1, 2, and 3 indicate that the temperature at which water should first evoke sensations of warmth lies at or below the presumed temperature of the 


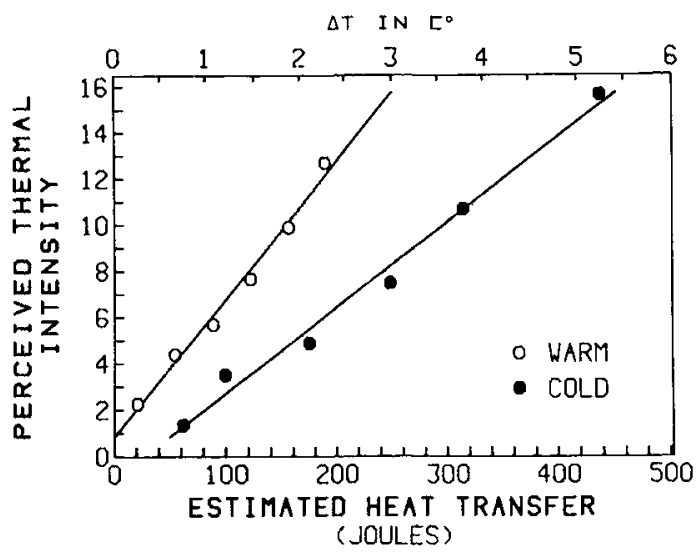

Figure 3. Perceived thermal intensity as a function of the estimated heat transfer between the liquid and the oral tissues. Note that for cold, direction of heat transfer is from tissue to liquid, whereas for warm it is from liquid to tissue. The corresponding changes in water temperature (positive for cold, negative for warmth) are shown along the top of the figure.

oral cavity. Projecting the best-fitting linear function for warmth (Figure 1) to zero (no sensation) predicts that the warmth threshold will occur at $34.9^{\circ} \mathrm{C}$, a full $2^{\circ}$ below body core temperature and $1^{\circ}$ below the temperature of most oral structures (Spierings, Peters, \& Plasschaert, 1984). In contrast, the same analysis for cold predicts that threshold will be reached at $31.9^{\circ} \mathrm{C}$, about $5^{\circ}$ below core temperature and $4^{\circ}$ below average oral temperature. If these projections are accurate, the mouth is remarkably more sensitive to small increments in heat than to small decrements in heat. The present experiment tested this hypothesis.

\section{Method}

Apparatus and Procedure. Thresholds for the perception of warmth and cold were measured for $20-\mathrm{ml}$ aliquots of water. The water stimuli were treated as in Experiment 1, except that the range of temperatures tested was changed to produce at most only weak sensations of warmth and cold. A pilot experiment established that thermal sensations from coolness through neutrality to warmth could be evoked with temperatures from $30^{\circ}$ to $36^{\circ} \mathrm{C}$ inclusive, at $1^{\circ}$ intervals (i.e., at $30^{\circ}$ and $36^{\circ} \mathrm{C}$, responses were $100 \%$ "cool" and $100 \%$ "warm", respectively). Stimulus delivery and expectoration were as before, with subjects again wearing gloves to eliminate nonoral sensations of temperature. During one half of the session, subjects held the water samples passively in the mouth (as in Experiment 1); during the other half, they swished the samples vigorously. These two conditions (their order counterbalanced across subjects) enabled evaluation of the effect of changing both the spatial configuration of the stimulus and the heat exchange between the water and the oral tissues. Swishing spreads the water more widely throughout the mouth and hence should heighten heat transfer.

The method of constant stimuli was used, in which each of the seven temperatures was presented three times in random sequence in both the "hold" and "swish" conditions. The intertrial interval was set at $30 \mathrm{sec}$, during which subjects were instructed to keep their mouths closed to promote the return to normal oral temperature. Subjects responded "cool," "'neutral," or "warm" immediately following expectoration of the stimulus, and were told to base their judgments upon the thermal sensation produced by the water in the mouth rather than upon an estimate of the temperature of the water relative to room temperature, body temperature, or any other thermal standard. ("Neutral" was included as a response category both to provide information about the range of perceived thermal indifference and to encourage relatively strict criteria for warmth and cold). Fifteen subjects contributed a total of 45 judgments per temperature in both conditions.

\section{Results and Discussion}

The results corroborate the estimates of threshold predicted from the suprathreshold data. Figure 4 displays the percentages of "cool," "neutral," and "warm" responses as a function of water temperature. If a criterion of $75 \%$ is chosen as the threshold level (i.e., point at which 3 of 4 stimuli would be called cool or warm), the cold threshold lies near $32^{\circ} \mathrm{C}$ and the warmth threshold near $35^{\circ} \mathrm{C}$. In fact, connecting the data points with straight lines and interpolating to the $75 \%$ response level produces estimates of threshold for the "hold" condition of $31.6^{\circ} \mathrm{C}$ for cold and $34.9^{\circ} \mathrm{C}$ for warmth. Recall that the suprathreshold functions predicted thresholds of $31.9^{\circ} \mathrm{C}$ and $34.9^{\circ} \mathrm{C}$. The agreement across psychophysical methods (given the $75 \%$ response criterion) supports the conclusion that the mouth is more sensitive to warming than to cooling.

Figure 4 also shows that swishing the water throughout the mouth fails to alter significantly the thresholds for warrnth and cold. The McNemar test of change applied to the data for $34^{\circ} \mathrm{C}$, where the largest differences occurred between conditions, showed no significance $\left(\chi^{2}(1)\right.$ $=2.25, \mathrm{p}<.25)$, and estimates of the "swish" thresholds, based upon extrapolation, were within $0.3^{\circ}$ of the "hold" thresholds.

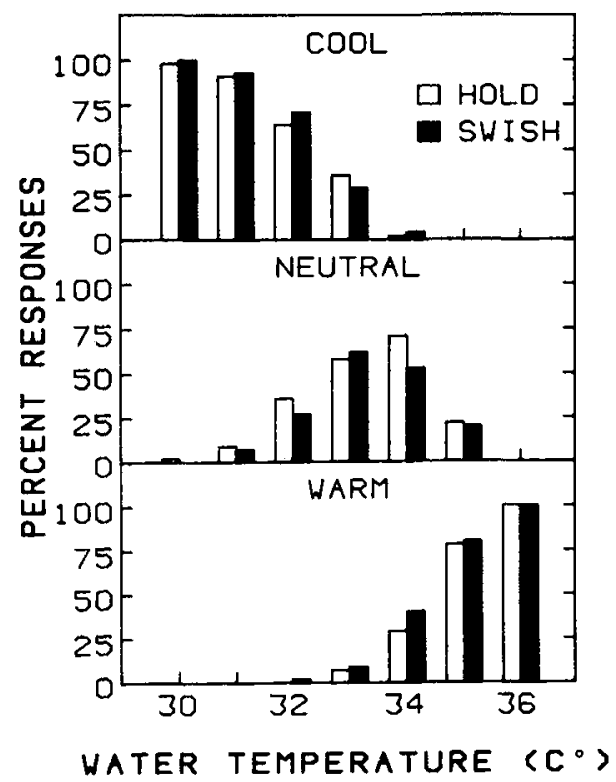

Figure 4. The percentages of "cool," "neutral," and "warm" responses as a function of initial water temperature for the "hold" and "swish" conditions. 


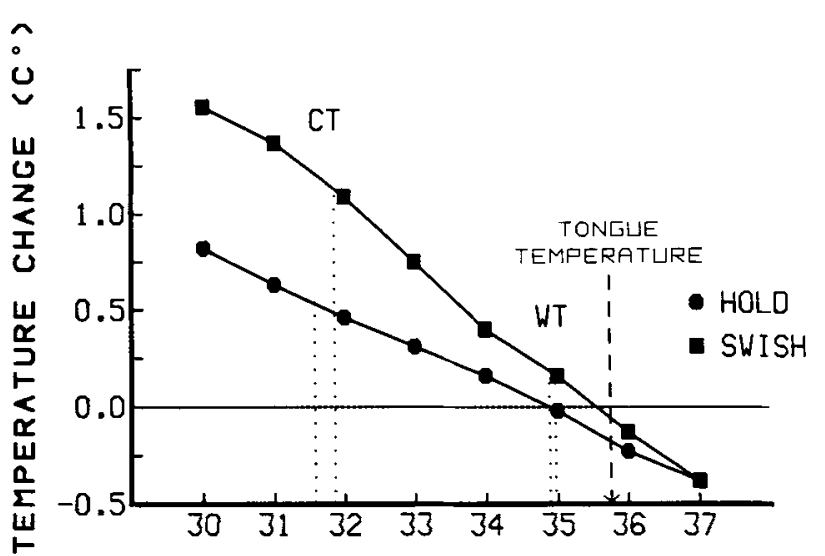

INITIAL WATER TEMPERATURE $\left(\mathrm{C}^{\circ}\right)$

Figure 5. The change in water temperature produced during sipping as a function of initial water temperature for the "hold" and "swish" conditions. Dotted lines extending from the functions to the abscissa denote the thresholds for cold (CT) and warmth (WT) calculated from the preceding psychophysical data. Tongue temperature was measured at the dorsal tip in the closed mouth (after $60 \mathrm{sec}$ ).

Figure 5 shows the change in water temperature that occurred at each temperature in the two conditions, along with the interpolated thresholds for warmth and cold. (Estimates of $\Delta \mathrm{T}$ were made as before, with the same two subjects). Swishing produced increasingly larger changes in water temperature at $35^{\circ} \mathrm{C}$ and below, but the cold threshold changed little. $\Delta \mathrm{T}$, and hence the estimated heat transferred from the tissue to the water, appears to be a poor predictor of the cold threshold. This may mean that judgments of cold arise during the initial moments of contact between the liquid and the oral tissue, when the temperature difference between the liquid and the tissue is at a maximum and the liquid is in contact with the anterior of the mouth. Subsequent swishing moves the liquid to other (probably less sensitive) oral areas while simultaneously causing its temperature to rise toward that of the tissue and saliva. The same is true for the warmth threshold, although the proximity of the warmth threshold to oral temperature meant that swishing could produce relatively little additional heat transfer near threshold (i.e., at the threshold for warmth, the liquid and the mucosa were near thermal equilibrium).

The location of the warmth threshold at a temperature roughly $1^{\circ}$ below the measured temperature of the oral tissues in the closed mouth seems paradoxical. How can a thermal stimulus produce a sensation of warmth when it fails to change skin temperature, or even cools the skin slightly? Based upon measurement of $\Delta \mathrm{T}$ for the water aliquots, the "hold" warmth threshold occurred when no heat exchange took place and the "swish" warmth threshold occurred when the water appeared to be marginally warmed (i.e., when heat was lost by the mucosa). At least three possible explanations exist. One is that the measurements of oral tissue temperature were erroneous; tissue temperature during the experiment may have fallen below $35^{\circ} \mathrm{C}$. This explanation was tested by measuring the temperature of the tonguetip, the area of the mouth so far established to be most responsive to warming (Green, 1984), $30 \mathrm{sec}$ after exposure to each of the water stimuli (presented randomly under the conditions of the experiment). This was done with the same two individuals from whom previous temperature measurements had been made. Mean tonguetip temperature (in the closed mouth) after $30 \mathrm{sec}$ was $35.5^{\circ} \mathrm{C}$, only a few tenths of a degree below the temperature of the tongue as measured in 15 subjects before exposure to water and after having the mouth closed for $60 \mathrm{sec}$ (shown in Figure 5 as the dashed line to the abscissa). Note that the poststimulation temperature was still above the threshold for warmth. It is notable that swishing produced no greater change in tongue temperature after $30 \mathrm{sec}$ than did simply holding the water passively in the mouth. The proximity of the warmth threshold to resting oral temperature is probably not an artifact of unusually low oral temperatures.

A second possibility is that subjects ignored the instructions and based their judgments on something other than the thermal sensation in the mouth, for example, on their estimates of how the water would feel to the fingers. That this probably did not occur was shown by a brief experiment in which subjects were told to judge the temperature of the water relative to the perceived temperature of the mouth itself, without reference to any other external standards. After 8 subjects showed the same pattern of responses as subjects in the main experiment, the measurements were terminated. Another brief test with 5 subjects also showed that, as at suprathreshold levels, a flavor (coffee) added to the water produced no changes in perceptions of warmth and cold. It therefore seems unlikely that the lower-than-expected thermal thresholds resulted from shifts in the criteria for warmth and cold caused either by the use of the extraoral standards or by past experience with the liquids.

A third explanation rests upon the observation (readily checked by introspection) that the closed mouth usually feels slightly warm. It follows that if a liquid fails to cool the mouth, a sensation of warmth will persist, and both the liquid and mouth will seem warm. This situation may exist because the resting temperature of the oral mucosa lies on the lower edge of the temperature range over which sensations of warmth never completely adapt (Kenshalo \& Scott, 1966), and where the rate of partial adaptation is slow (Kenshalo, 1970). Thus, a 30-sec intertrial interval was undoubtedly too short to ensure that adaptation had progressed maximally; yet maximal adaptation may still have left lingering sensations of warmth. The high resting temperature of the oral cavity apparently has the effect of biasing the mouth to perceive as warm no change or very small increases in skin temperature, whereas small decreases in skin temperature produce either a reduction in warmth sensation (Kenshalo, 1970) or the perception of thermal neutrality.

Additional confirmation of the validity of the threshold values is given in Figure 6, where the data of Experi- 


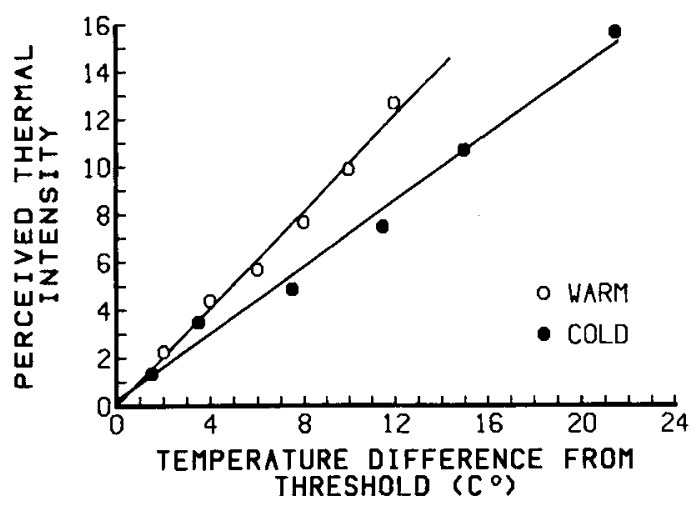

Figure 6. The data of Figures 1 and 2 for deionized water replotted in terms of the threshold for warmth and cold measured in Experiment 2. Convergence of the best-fitting functions at $(0,0)$ indicates agreement between threshold and suprathreshold measurements of thermal sensation.

ment 1 are replotted relative to the thresholds for warmth and cold. The best-fitting linear functions intercept the $y$-axis near zero and show that perceived warmth grows more rapidly than perceived cold (slopes $=1.01$ and 0.69 , $t(15)=1.95, p<.05)$. The divergence in the functions indicates that the difference in thermal intensity between modalities increases as temperatures become more extreme.

\section{GENERAL DISCUSSION}

This study has shown that when thermal stimulation occurs over large areas of the oral mucosa, the mouth is a better sensor of warming than of cooling. An analysis of oral heat-sensing capacity demonstrated that sensations of warmth arise in the presence of little of no heat transfer, whereas liquids must be as much as $5^{\circ} \mathrm{C}$ below the temperature of the oral mucosa to be always perceived as cool.

The relatively high temperature of the oral mucosa may be the primary factor underlying the unexpectedly low oral thresholds for warmth. It has been shown on external skin that whether or not a given change in skin temperature evokes a threshold-level thermal sensation depends upon the resting temperature of the skin (Kenshalo, 1970). This implies that if oral temperatures were lower, the threshold for warming would correspond to a larger $\Delta \mathrm{T}$, and the threshold for cooling to a smaller $\Delta \mathrm{T}$. This hypothesis is not easily tested, however, because no amount or frequency of oral rinses produces a stable shift in oral temperature. As reported by Pangborn, Chrisp, \& Bertolero (1970), repeated cold-water rinses lower average oral temperature, but rapid shifts in temperature occur immediately upon expectoration of the "adaptor" rinse. Such thermal transients would introduce an unacceptable confound into threshold measurements.

That the oral psychophysical function for warmth generated by liquids has a steeper slope than the psychophysical function for cold is consistent with the results of J. C. Stevens and S. S. Stevens (1960), who carried out the first quantification of thermal perceived magnitudes on external skin. The oral cavity is therefore not unique in this respect; the human perceptual response to warming appears in general to grow more rapidly for a given change in skin temperature than does the response to cooling. This may be a consequence of the fact that the range of temperatures between threshold and heat pain is smaller than the range between threshold and cold pain. Because the warmth threshold occurs in the region of $30^{\circ}-35^{\circ} \mathrm{C}$ on various body loci, an increase of only $10^{\circ}$ to $15^{\circ} \mathrm{C}$ can produce sensations of pricking pain (Hardy, H. G. Wolff, \& Goodell, 1952). In contrast, cooling the surface of the skin to near $0^{\circ} \mathrm{C}$ (by submerging the hand in a water bath) may not cause pain during a 10 - to 15 -sec exposure, even though this constitutes a $\Delta \mathrm{T}$ of perhaps $30^{\circ} \mathrm{C}$ (Wolff \& J. D. Hardy, 1941). The high temperature of the mouth should maximize this difference between the perceptual ranges of warmth and cold.

The present results demonstrate once again that thermal responsiveness to small stimuli is a poor predictor of responsiveness to larger stimuli (see J. C. Stevens \& Green, 1978). It was found in this laboratory (Green, 1984) that punctiform maps of oral sensitivity correlate well with suprathreshold responses to larger temperature probes, but that some areas virtually devoid of warm spots nevertheless exhibit some responsiveness to warming. In the present case, the greater spatial heterogeneity of oral warmth responsiveness than of cold responsiveness failed to predict the superior sensitivity (and suprathreshold responsiveness) of the whole mouth to warming. Both of these predictive failures probably owe much to the presence of spatial summation in the thermal senses (J. C. Stevens \& Marks, 1971, 1979), and illustrate the fundamental importance of summation in temperature perception. The experience of temperature produced by liquids in the mouth is characterized more by uniformity than by heterogeneity, and only with careful introspection can we begin to appreciate regional differences in sensory intensity. Future studies should perhaps include analyses of the relative contributions of the different oral regions to the overall summated sensation of temperature in the mouth. Of particular interest may be the teeth, whose influence upon the perception of the temperature of liquids may range from insignificant in the mid-range of temperatures to virtually dominant at the coldest temperatures.

\section{REFERENCES}

Green, B. G.(1984). Thermal perception on lingual and labial skin. Perception \& Psychophysics, 36, 209-220.

Hardy, J. D. Wolff, H. G., \& Goodell, H. (1952). Pricking pain threshold in different body areas. Proceedings of the Society of Experimental Biology and Medicine, 80, 425-427.

HiRsCh, V. L., \& Schriever, H. (1929). Beitrag zur Sensibilitat der Zunge des Kehlkopfes und der hinteren Rachewand. Zeitschrift für Biologie, 89, 1-20.

KenshaLo, D. R. (1970). Psychophysical studies of temperature sensitivity. In W. D. Neff (Ed.), Contributions to sensory physiology (Vol. 4, pp. 19-74). New York: Academic Press.

Kenshalo, D. R., \& ScotT, H. A., JR. (1966). Temporal course of thermal adaptation. Science, 151, 1095-1096. 
Pangborn, R. M., Chrisp, R. B., \& Bertolero, L. L. (1970). Gustatory, salivary, and oral thermal responses to solutions of sodium chloride at four temperatures. Perception \& Psychophysics, 8, 69-75.

Spierings, Th. A. M., Peters, M. C. R. B., \& Plasschaert, A. J. M. (1984). Surface temperature of oral tissues: A review. Journal de Biologie Buccale, 12,91-99.

Stevens, J. C., \& Green, B. G. (1978). History of research on feeling. In E. C. Carterette and M. P. Friedman (Eds.), Handbook of perception (Vol, 6, pp. 3-25). New York: Academic Press.

Stevens, J. C., \& MARKS, L. E. (1971). Spatial summation and the dynamics of warmth sensation. Perception \& Psychophysics, 9, 391-398.

Stevens, J. C., \& Marks, L. E. (1979). Spatial summation of cold. Physiology \& Behavior, 22, 541-547.

Stevens, J. C., \& Stevens, S. S. (1960). Warmth and cold: Dynamics of sensory intensity. Joumal of Experimental Psychoiogy, 60, 183-192.
WolfF, S., \& HARDY, J. D. (1941). Studies on pain: Observations on pain due to local cooling and on factors involved in the "cold pressor" effect. Joumal of Clinical Investigation, 20, 521-533.

\section{NOTES}

1. No systematic attempt was made to measure changes in tissue temperature during exposure to water because of the uncertainty of the accuracy of such measurements, and because no single measure of oral temperature can be considered representative of the whole mouth (Spierings et al., 1984).

(Manuscript received May 20, 1985; revision accepted for publication November 25,1985 .) 